\title{
Postnatal Change in Inhibitory Regulation of Intestinal Motor Activity in Human and Canine Neonates ${ }^{1}$
}

\author{
JOHN BAKER AND CAROL LYNN BERSETH \\ Department of Pediatrics [J.B.] and Department of Pediatrics and Gastroenterology Unit [C.L.B.], Mayo \\ Clinic, Rochester, Minnesota 55905
}

\begin{abstract}
Motor activity was recorded in 19 preterm infants three times during the first postnatal month. There was a paucity of small intestinal motor quiescence during fasting in the first postnatal week; however, its duration significantly increased with postnatal age $(p<0.03)$. Although fasting motor patterns changed with postnatal age, motor responses to feeding were present within the first few days of life. Motor activity was also recorded weekly in 11 newborn dogs for $6 \mathrm{wk}$. Intestinal motor quiescence was also absent during fasting for the first postnatal week but it significantly increased with postnatal age $(p<0.002)$. As in the human preterm infant, a motor response to feeding was present within the first few days of life. Plasma concentrations of gastrin and peptide YY during fasting were low in the preterm human and canine neonate during the first postnatal week but plasma concentrations of both peptides increased with postnatal age. Although plasma concentrations of gastrin were low during fasting for the first postnatal week, plasma concentrations of gastrin increased significantly postprandially compared with fasting ( $p$
\end{abstract}

ABSTRACT
Adequate enteral nutrition is vital to the long term survival of premature infants. Although mucosal functions of the small intestine are adequate to support the use of enteral nutrition by 28-32 wk of gestation, intestinal motor function lags behind (1). Motor activity is responsible for the aboral movement of nutrients through the gastrointestinal tract. During fasting healthy children and adults demonstrate a cyclical pattern of motor activity. The gut displays motor quiescence that is replaced by episodes of irregular contractions, which, in turn, are replaced by intense phasic contractions that migrate aborally. This entire cycle of motor activity, called the interdigestive cycle, occurs approximately every $60-90 \mathrm{~min}$. When the adult eats, this cycling pattern is interrupted by a pattern of continuous contractile activity. This change in motor activity is called the "fed response."

Received June 6, 1994; accepted February 5, 1995

Correspondence and request for reprints: C. L. Berseth, M.D., Department of Pediatrics/ Newborn Section, Baylor College of Medicine, One Baylor Plaza, Houston. TX 77030.

Supported by Grant No. HD 24558 from the National Institutes of Health

1 Presented in part before the Society for Pediatric Research in Baltimore, Mav 5. 1992
$<0.05$ ). We conclude that motor quiescence during fasting becomes a more prominent feature of newborn intestinal motor function postnatally. In addition the release of two peptides that regulate motor patterns also change postnatally. Thus, postnatal changes in motor patterns and peptide release change in a parallel fashion in human preterm neonates and canine neonates. We speculate that the postnatal change in intestinal motor quiescence reflects changes in inhibitory regulation by the enteric nervous system and that the neonatal dog will provide an excellent animal model to explore the mechanisms that regulate maturation of small intestinal motor function in the preterm infant. (Pediatr Res 38: 133-139, 1995)

PYY, peptide YY;

Abbreviations

ENS, enteric nervous system

MMC, migrating motor complex

In contrast to these motor patterns seen in adults and children, complete interdigestive cycles are rarely present in preterm infants $(2,3)$. However, interdigestive cycles appear postnatally if the infant receives routine enteral feedings $(4,5)$. As in the preterm human, small intestine motor activity patterns also change postnatally in the term neonatal dog (6). Small intestinal motor activity consists of irregular spiking activity at birth, migrating activity appears by $2 \mathrm{wk}$, and the adult interdigestive cycle is established by 6 wk (6). Although previous studies have described the maturation of motor activity in the human and canine neonate, none has evaluated the appearance of motor quiescence in the maturing newborn gut. Because adult feeding disorders are frequently associated with the loss of inhibitory regulation of motor activity, we speculated that the postnatal changes in motor activity that occur in human neonates may reflect and/or occur simultaneously with changes in motor quiescence. Motor activity in the adult is regulated by neural and hormonal input. Gastrin, for example, is an excitatory peptide, whereas PYY is inhibitory. Plasma concentrations of these gut peptides that modulate motor ac- 
tivity differ in preterm infants and adults. Plasma concentrations of gastrin in preterm infants are approximately $50 \%$ lower than in adults (7), and plasma concentrations of PYY in the preterm infant exceed adult values by $2-5$-fold (8). Plasma concentrations of gastrin in neonatal dog also change with age (9), but the postnatal release of these peptides has not been fully characterized in the human or the canine neonate. Because medical and ethical concerns limit investigational studies in infants, an animal model that reproduces the postnatal changes of intestinal motor patterns seen in preterm infants as well as the hormonal and neural component of its regulation would permit further study of the mechanisms that regulate motor activity patterns in preterm human infants. The purpose of this study was to evaluate whether functional maturation of neonatal dog intestine parallels that of the preterm human infant with respect ot motor patterns and release of gastrointestinal peptides that modulate motor patterns.

\section{METHODS}

Subjects. Studies in infants were approved by the Mayo Clinic and Foundation Institutional Review Board. Nineteen preterm infants who ranged 29-32 wk in gestational age participated in this study. All had been admitted to the Newborn Intensive Care Unit (NICU) at St. Mary's Hospital for ventilator support for respiratory distress syndrome. To study a relatively healthy population, we excluded infants who required ventilator support longer than $5 \mathrm{~d}$ or who had a diagnosis of intraventricular hemorrhage or patent ductus arteriosus. None of the infants had received enteral nutrition while they required ventilator support, and all infants entered the study on the day the neonatologist had chosen to begin enteral nutrition. All parents provided informed consent for their infant's participation in this study. All infants were studied at three postnatal ages: d 0-4 (wk 1), d 10-14 (wk 2), and d 24-30 (wk 4). After entry into this study, all infants received routine enteral feeding throughout their NICU hospitalizations. No infant had received enteral feedings before their first serial study. After the completion of this first study, infants were fed $24 \mathrm{~mL} / \mathrm{kg} / \mathrm{d}$. Thereafter the volume was increased by $24 \mathrm{~mL} /$ $\mathrm{kg} / \mathrm{d}$, and the parenteral nutrition decreased accordingly until a maximal enteral volume of $150 \mathrm{~mL} / \mathrm{kg} / \mathrm{d}$ was achieved and maintained. Thus, most infants had achieved full enteral nutrition by postnatal wk 2 . All infants were initially fed a 20 calories/oz formula until full enteral nutrition was achieved; then they were given a 24-calories/oz formula (Similac and Similac Special Care; Ross Products Div., Columbus, OH).

Studies in dogs were approved by the Mayo Clinic and Foundation Animal Care and Use Committee. Eleven pups from four litters of mixed breed hounds were studied. All pups remained with their mothers to nurse; all survived and gained weight. Pups that were runted had congenital anomalies or that developed an infection during the first postnatal week were excluded from the study. Manometric and peptide studies were performed weekly the first $6 \mathrm{wk}$ of life.

Manometry. Motor activity was recorded using our neonatal low compliance, continuous perfusion system previously reported $(2,10)$ and validated (2). This system provides an infusion rate of $0.01 \mathrm{~mL} / \mathrm{min} /$ recording port with a response rate of $57 \mathrm{~mm} \mathrm{Hg}$ at 10 pounds/square inch.

For studies performed in infants, this system was used to perfuse a manometric tube that was positioned such that all recording ports were beyond the pylorus. This tube, which was made of polyvinyl extrusion tubing in the Mayo Clinic Gastroenterology Unit, had an outer diameter of $3.5 \mathrm{~mm}$, and contained four manometric ports spaced $2.5 \mathrm{~cm}$ apart. On each study day, an infant was fasted for $2 \mathrm{~h}$ and placed without sedation supine with the right side down. Fasting was limited to $2 \mathrm{~h}$ based on our previous studies showing that feeding responses do not persist beyond $2 \mathrm{~h}$ (11). While the tube was actively perfused, the recording ports were noted to pass from antrum and into the duodenum as the frequency of phasic contractions changed from three contractions/min, to one of nine contractions/min. Motor activity was recorded for $4 \mathrm{~h}$. Then each infant received a feeding infusion over $15 \mathrm{~min}$ with $10 \mathrm{~mL} / \mathrm{kg}$ of Similac formula (Ross) via a separate second orogastric tube. Motor activity was recorded throughout this feeding and for $2 \mathrm{~h}$ after its completion.

In dogs, the manometric system perfused a manometric tube that was placed with fluoroscopic guidance. This tube, also made in the Mayo Clinic Gastroenterology Unit from polyvinyl extrusion tubing, had an outer diameter of $4.0 \mathrm{~mm}$ and contained six recording ports spaced $2.5 \mathrm{~cm}$ apart. On study days, pups were removed from the mother for $2 \mathrm{~h}$. For tube placement the unsedated pup was placed on its back with the right side down and the tube placed under fluoroscopy. The pup was then placed prone for the remainder of the study, and the tube was taped in place. After the manometric tube was placed, motor activity was recorded in unsedated pups for 2-h fasting. Then a second orogastric tube was placed, and each pup received a gastric feeding infusion over $15 \mathrm{~min}$ with $10 \mathrm{~mL} / \mathrm{kg}$ of Esbilac formula (Carnation, Hampshire, IL), a standard neonatal formula for pups. Motor activity was recorded throughout this feeding and for $2 \mathrm{~h}$ after its completion.

Gastrointestinal peptide concentrations. In human infants 1.2-mL blood samples were obtained from indwelling central catheters. While the manometric studies were being performed during wk 1, 2, and 4, a single blood sample was drawn from a central arterial catheter after $4 \mathrm{~h}$ of fasting. During wk 1 and 2 , additional blood samples were drawn at 30 and $60 \mathrm{~min}$ after the initiation of the feeding. In dogs, fasting blood samples were drawn weekly by venipuncture after pups remained away from the mother for $4 \mathrm{~h}$. During wk 1 and 2, additional blood samples were drawn 15 and $30 \mathrm{~min}$ after the completion of the bolus feeding infusion.

All blood samples were placed on ice in heparinized tubes and transported to the laboratory. Plasma was stored at $-70^{\circ} \mathrm{C}$, and all samples were processed in a single assay. Concentrations of gastrin and PYY were determined in duplicate samples using established RIA $(7,8)$.

Data analysis. Motor activity was analyzed in 30-min intervals. The presence of motor quiescence was identified and characterized as follows. First, motor activity was defined to be present when three or more contractions exceeding $6 \mathrm{~mm} \mathrm{Hg}$ occurred within a 1-min interval. Second, any high amplitude contraction occurring simultaneously in three or more leads 
A

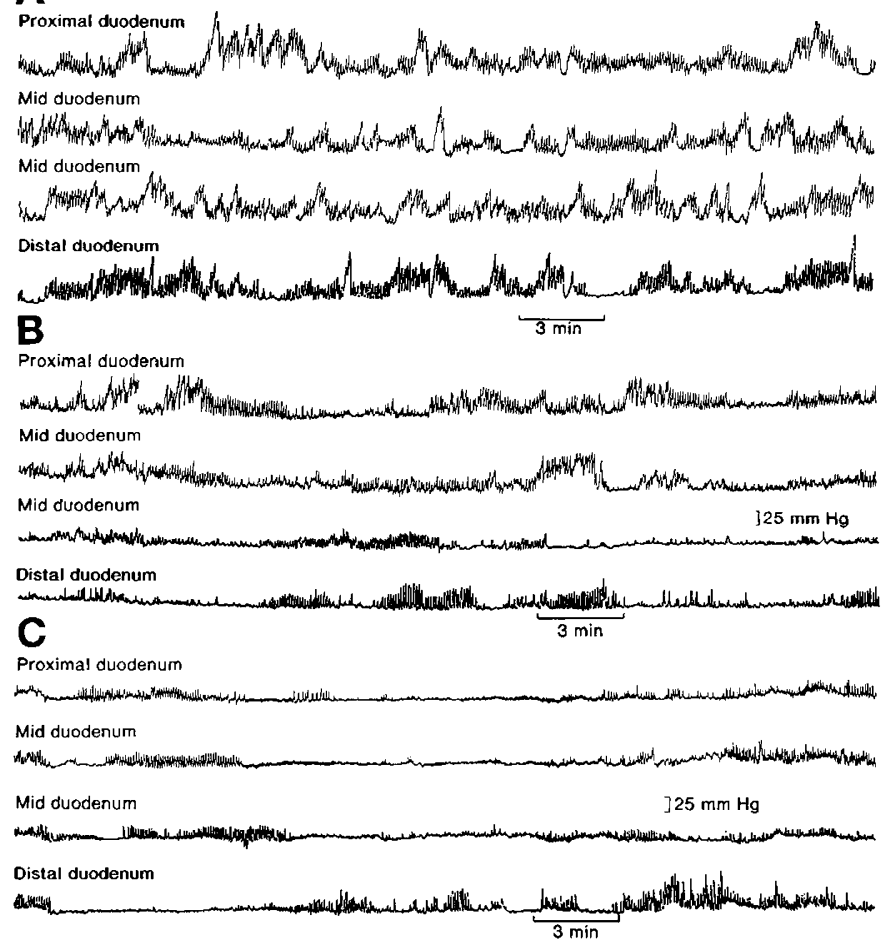

Figure 1. Motor activity during fasting in a preterm infant during $(A)$ postnatal wk $1,(B)$ postnatal wk 2 , and $(C)$ postnatal wk 4 . The top most line of recording demonstrates motor activity recorded from the proximal duodenum. Each lower line represents motor activity recorded $2.5 \mathrm{~cm}$ distal to the line above it. During the first postnatal week $(A)$, note that phasic contractions occupy most of the recording and that very little motor quiescence is present. In this same infant 2 wk later $(B)$ phasic contractions are "clustered" together and separated by episodes of motor quiescence that range 1-3.5 min in duration. In this same infant at $4 \mathrm{wk}$ postnatal age $(C)$, motor quiescence is pronounced, with episodes that range from 0.5 to $11 \mathrm{~min}$ in duration, and phasic contractions now migrate distally in a sequential manner.

was identified to be an artifact due to movement. All other portions of the recording that did not contain a movement artifact or motor activity were identified to contain motor quiescence. When motor quiescence was present, its overall duration was calculated in $\mathrm{min} / \mathrm{h}$ of recording. Because each subject provided repeated samples and analysis of variance could not be performed, a regression line for each subject was determined as a function of postnatal age. The mean of those regression lines was compared with 0 by unpaired $t$ testing.

Plasma peptide concentrations were expressed in $\mathrm{pg} / \mathrm{mL}$. Fasting and postprandial concentrations in individual animals were compared by analysis of variance for repeated measures followed by a multiple comparison procedure (least squares difference). In addition, postprandial release of peptide was calculated as the area under the curve using the trapezoid rule; values for wk 2 were compared with those for wk 0 by paired testing.

\section{RESULTS}

Motor activity. The patterns of motor quiescence and activity that occurred during fasting in the preterm infant changed with postnatal age. During wk 1 infants displayed continuous contractile activity that was interrupted by little or no motor
A
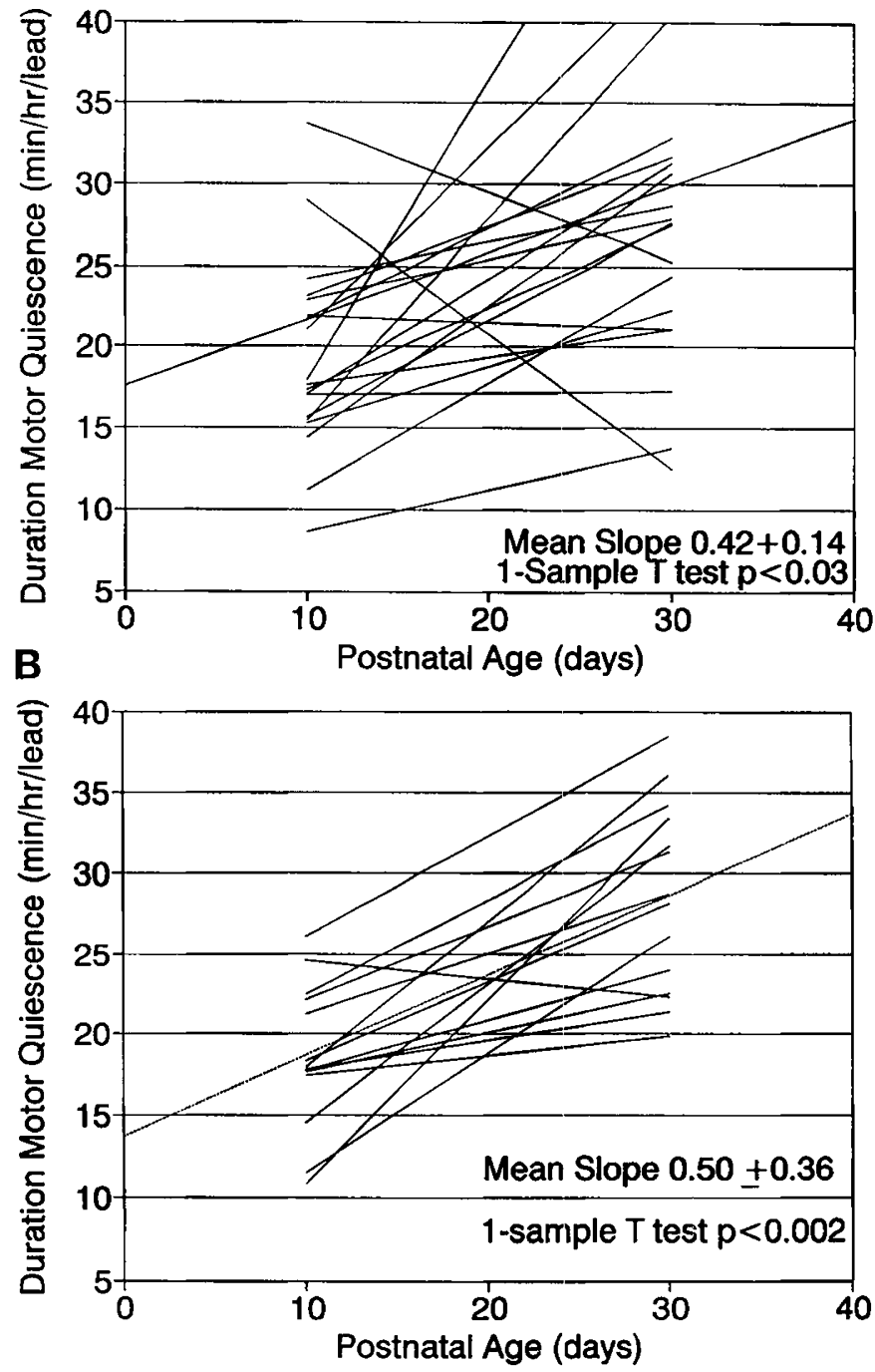

Figure 2. Regression analysis of motor quiescence duration as a function of postnatal age in $(A)$ human infants and $(B)$ canine neonates. Individual lines are shown by the hatched lines and the composite line by a solid line.

quiescence (Fig. 1A). By wk 2 short episodes of motor quiescence alternated with episodes of clustered phasic contractions (Fig. 1B). Finally, by wk 4, episodes of motor quiescence were more prominent, and clustered phasic contractions appeared to migrate sequentially aborally (Fig. $1 C$ ). The duration of motor quiescence significantly increased with postnatal age (Fig. 2A; $p<0.03$ ). In spite of the presence of these postnatal changes in motor quiescence, motor activity changed in response to feeding within the first few days of life (Fig. 3A). During the first postnatal week the continuous motor activity seen during fasting was replaced by persisting motor quiescence of approximately 30-min duration. Thereafter the pattern of persisting motor activity recurred. By the second week, the pattern of alternating episodes of motor quiescence and phasic contractions was replaced by one of persisting motor quiescence of approximately 30-min duration. This prolonged episode of motor quiescence was then replaced, in turn, by one of uninterrupted contractile activity of approximately 30 -min duration. Thereafter, the fasting pattern of alternating quiescence and phasic contractions resumed. 


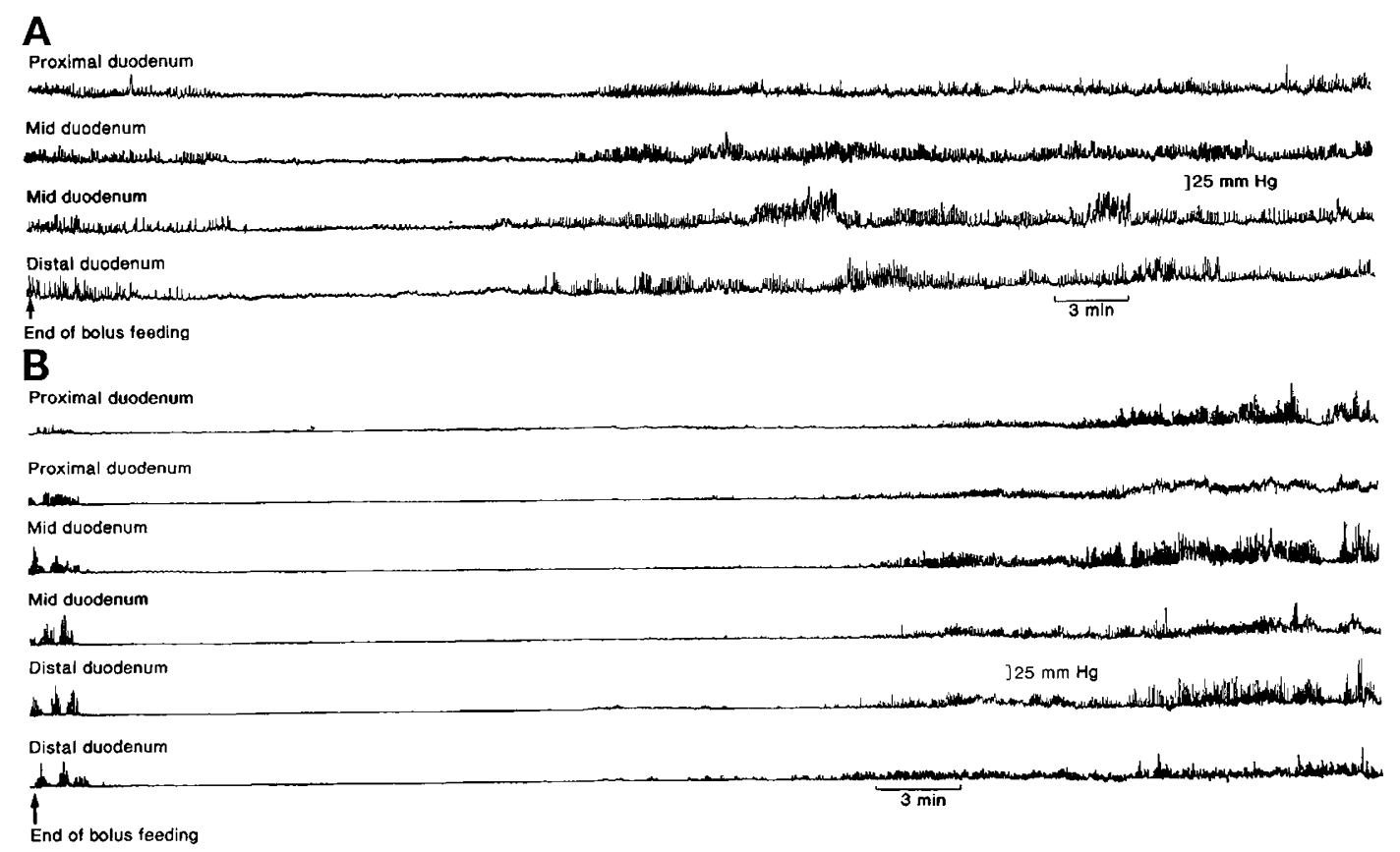

Figure 3. Motor activity in response to an orogastric feeding in $(A)$ a human neonate born at 30-wk gestation and $(B)$ a canine neonate at 1-wk postnatal age. The orientation and magnification is similar to those in Figure 1. Note that, in the human, with the onset of the feeding the typical pattern of alternating phasic contractions and motor quiescence is initially replaced by an episode of motor quiescence that is sustained for approximately 30 min. In turn, motor quiescence is replaced by persisting contractions for approximately $30 \mathrm{~min}$. Thereafter, the fasting pattern of alternating episodes of phasic contractions and motor quiescence resumes. Similarly in the pup $(B)$ the typical fasting pattern of alternating episodes of phasic contractions and motor quiescence is replaced by motor quiescence with the initiation of the feeding. Motor quiescence, which persists for approximately 30 min, is replaced, in turn, by persisting contractions.

As in the human, the pattern of motor quiescence changed with postnatal age in the neonatal dog. During fasting puppies displayed continuous contractile activity that was rarely interrupted by the presence of motor quiescence (Fig. $4 A$ ). By wk 2 motor quiescence was more apparent and was interspersed among episodes of phasic contractions (Fig. $4 B$ ). By wk 5 motor quiescence was prominent and episodes of phasic contraction appeared to migrate distally (Fig. 4C). As in preterm humans, the duration of motor quiescence in neonatal dogs increased significantly as a function of postnatal age (Fig. $2 B$; $p<0.002$ ).

In response to a feeding, canine intestinal motor activity changed in a manner similar to that seen in human infants. With the initiation of the feeding during the first week, the pattern of persisting motor activity was replaced by one of motor quiescence of approximately 30-min duration. By the second week the pattern of alternating episodes of motor quiescence and phasic contractile activity was replaced by one of prolonged motor quiescence (Fig. $3 B$ ). Motor quiescence persisted for approximately $30 \mathrm{~min}$ and was subsequently replaced by persistent contractile activity. Persistent contractile activity was present for approximately $30 \mathrm{~min}$; then the alternating pattern of motor quiescence and phasic contractile activity similar to that seen during the prefeeding period resumed.

Plasma gastrin and PYY. In the preterm infant plasma concentrations of gastrin during fasting increased significantly at postnatal wk 2 and 4 and plasma concentrations of PYY increased significantly at wk 4 compared with wk 1 (Figs. 5 and 6). Despite the presence of lower plasma gastrin concen- trations during the first postnatal week, plasma concentration values increased significantly in response to a feeding ( $p<$ 0.05 compared with fasting). Although the overall gastrin release in response to feeding was greater during wk 2 compared with 1 (7147 versus 1035), the variance was too large to detect a difference. Postprandial plasma concentrations of PYY did not differ from those during fasting.

Similar to the postnatal rise in fasting gastrin in human neonates, plasma concentrations of gastrin during fasting in neonatal dogs increased during postnatal wk 3-6 compared with birth (all $p<0.03$, compared with fasting). Fasting plasma concentration of PYY increased significantly at wk 1 and 2 compared with those seen at birth and then declined to values similar to those for birth (Fig. 7). Similar to the preterm infants, a significant release in the neonatal canine gastrin occurred during the first week in response to feeding ( $p<$ 0.05 , compared with fasting). Moreover overall gastrin release was greater during wk 2 than wk 1 (2798 versus 1025; $p<$ 0.01). Postprandial plasma concentrations of PYY were similar to those during fasting during wk 1 and 2.

\section{DISCUSSION}

Although the postnatal change in motor activity has been characterized previously in human infants (3), the focus of this study was motor quiescence. In the healthy adult, motor quiescence occupies $40-60 \%$ of the recordings of intestinal motor activity (12). Although motor activity patterns are often abnormal in adults who have feeding disorders, differences in the occurred and/or organization of motor quiescence are also 
A

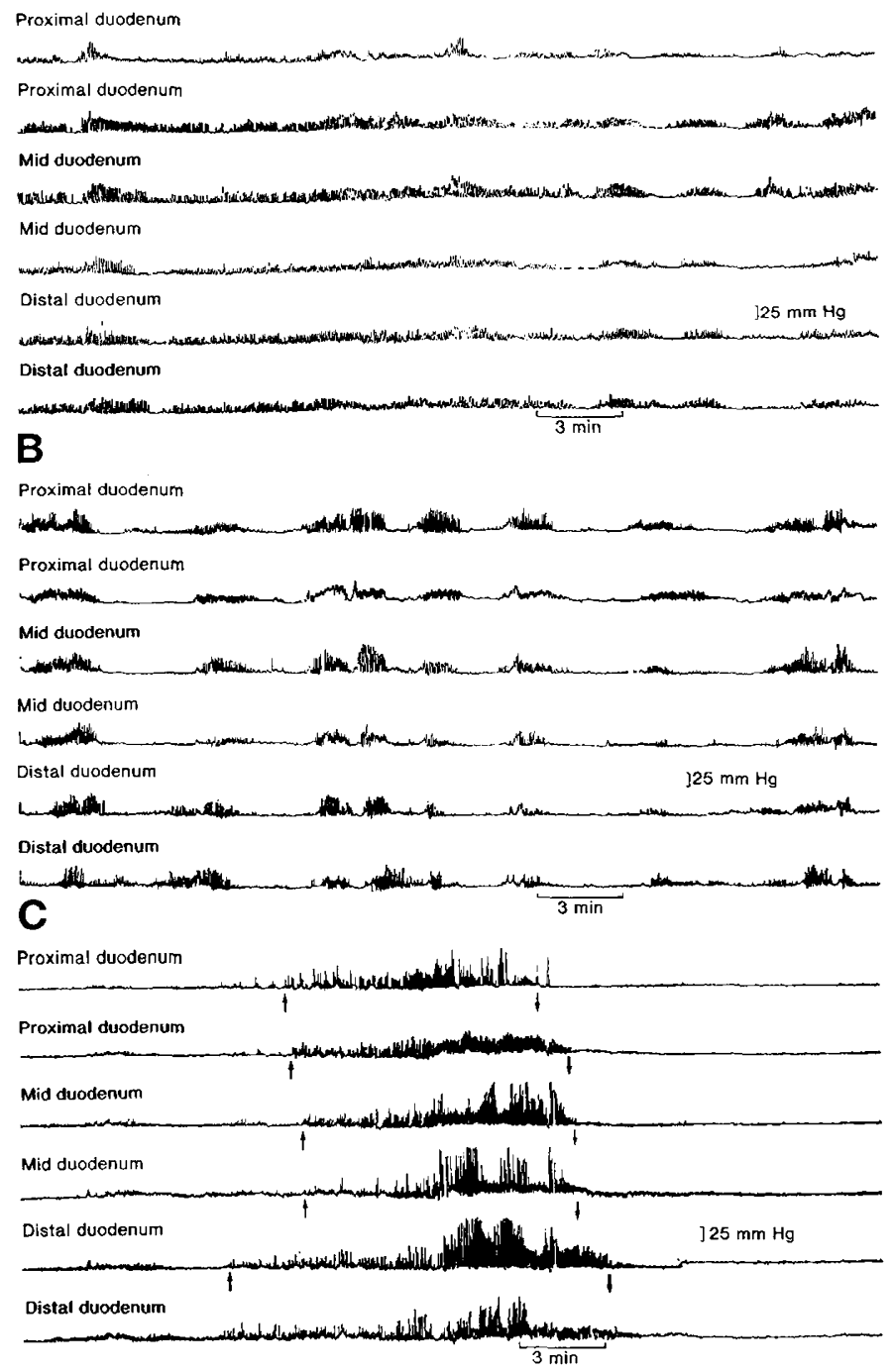

Figure 4. Motor activity during fasting in a canine neonate during $(A)$ postnatal wk $1,(B)$ postnatal wk 3 , and $(C)$ postnatal wk 6 . The orientation and magnification is similar to those in Figure 1 . Note that phasic contractions occupy most of the recording and that little motor quiescence is present during the first postnatal week $(A)$. By wk $3(B)$, this same pup now displays phasic contractions that are "clustered" and separated by episodes of motor quiescence that range 1-4 min in duration. By postnatal wk $6(C)$ prolonged episodes of motor quiescence are present and phasic activity migrates from proximal to distal duodenum. Upward directed arrows indicate the initiation of migratory activity and downward directed arrows the termination of the migration.

observed (12). Although the maturation in motor quiescence discussed here may reflect maturation of cycling activity, most of the infants in this study did not display MMC. Thus, we speculate that organization of motor quiescence occurs as a phenomenon independent of that of migrating activity. Inhibitory regulation of motor activity is exerted by the ENS, and loss of ENS regulation in adults with diabetic neuropathy frequently results in feeding intolerance (12). Milla has speculated that gestational changes in human neonatal motor activity patterns reflect changes in ENS maturation (3). In our neonatal canine model, markers for ENS maturation mature postnatally in concert with changes in motor quiescence (13). Furthermore, the absence of motor quiescence in the human

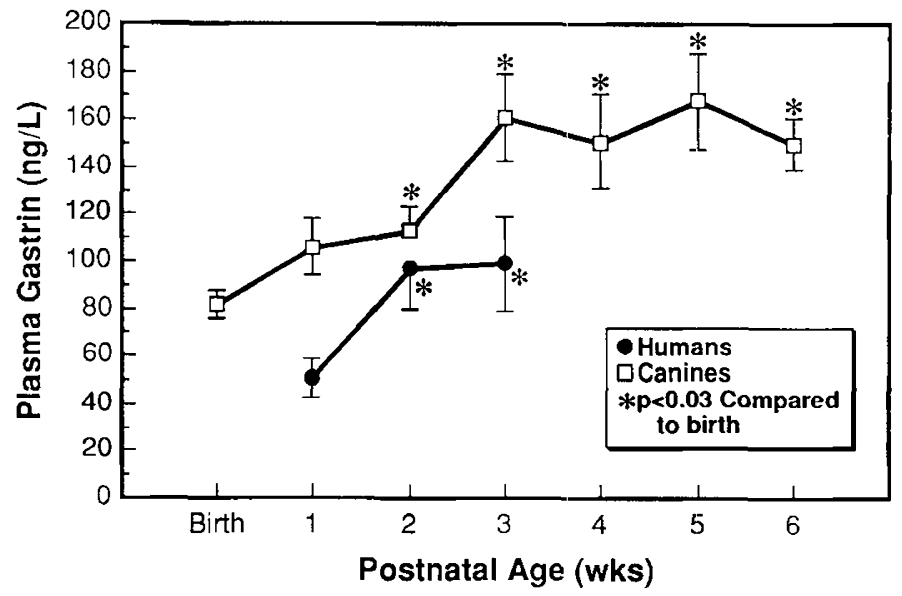

Figure 5. Postnatal changes in fasting plasma gastrin concentration in human and canine neonates. Values are shown for humans for postnatal wk 1, 2, and 4 and designated by solid circles. Values for pups are shown for wk $0-7$ and designated by open squares. All values are shown as the mean \pm SEM.

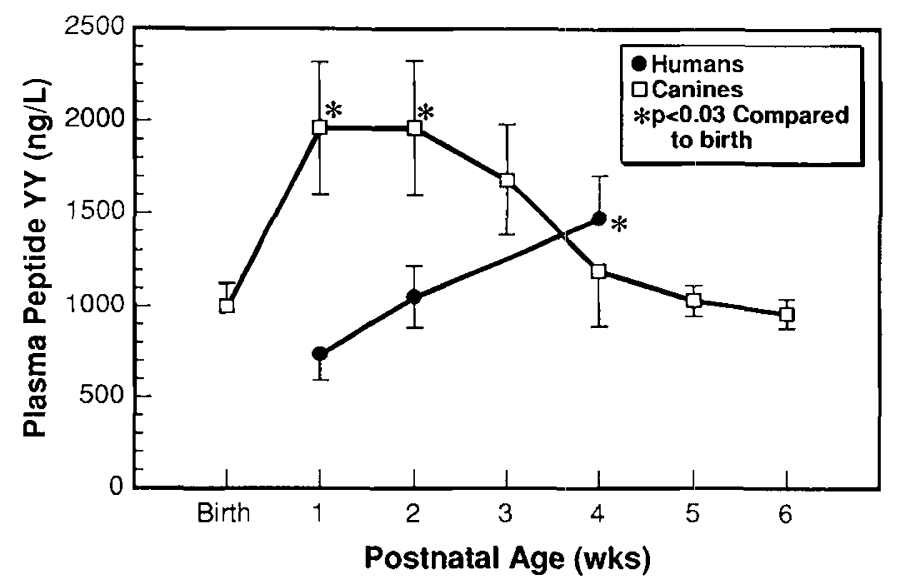

Figure 6. Postnatal changes in fasting plasma PYY concentration in human and canine neonates. Values are shown for humans for postnatal wk 1, 2, and 4 and designated by solid circles. Values for pups are shown for wk $0-7$ and designated by the open squares. All values are shown as the mean $\pm \mathrm{SEM}$.

preterm infant is predictive of feeding intolerance (14). The current study confirms that significant changes in motor quiescence occur in the maturing neonatal small intestine in concert with the changes in contractile activity previously described (10).

In a previous study in human infants, we serially monitored plasma concentrations of peptides that are released as nutrients enter sequentially more aboral sites in the gastrointestinal tract (8). By using this indirect measuring technique we demonstrated that aboral movement of nutrients occurs despite the absence of MMC (8). Thus, the pattern of alternating episodes of motor quiescence and phasic contractions must achieve aboral transit in the preterm infant and newborn dog. Just as lack of ventricular filling time can impair cardiac output, we speculate that the lack of motor quiescence in the newborn gut may contribute significantly to the impairment of aboral movement of nutrients in the preterm intestine.

Interestingly, motor quiescence replaced phasic activity at birth in response to feeding. The portion of the fed response occupied by motor quiescence, however, decreases with post- 


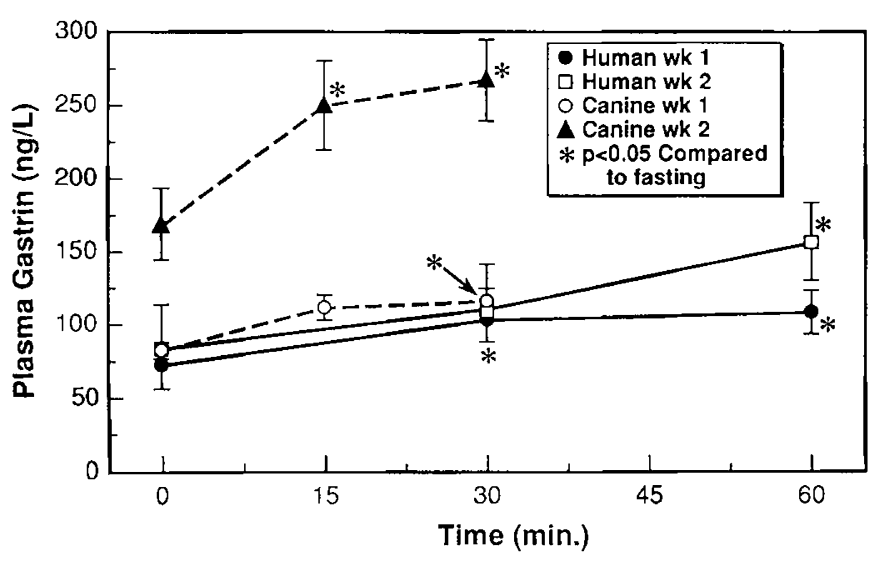

Figure 7. Fasting and postprandial plasma concentrations of gastrin in human and canine neonates. Values for humans during wk 1 are shown by solid circles and for wk 2 by the open squares. Values are shown for times $0,30,60,150$, and $180 \mathrm{~min}$ after the initiation of a 2 -h continuous infusion. Values for pups during wk 0 are shown by the open circles and during wk 2 by the solid triangles. Values during times 0,30 , and $60 \mathrm{~min}$ after a bolus infusion are shown.

natal age in dogs (15), suggesting that there is a loss of inhibitory regulation in response to feeding with age. Recent studies in human preterm infants now confirm that this same postnatal loss of inhibitory regulation of the fed response occurs in the preterm infant (13). Subjects in this study were fed $10 \mathrm{~mL} / \mathrm{kg}$. Thus, most were given a volume that was much smaller than their routine intake by study 3 . However, all subjects were fed $10 \mathrm{~mL} / \mathrm{kg}$ at all study points to standardize the treatment. Moreover, we have previously shown that volumes as low as $4 \mathrm{~mL} / \mathrm{kg}$ elicit normal feeding responses (11). Collectively these studies suggest that different regulatory mechanisms control intestinal motor activity during feeding and fasting, but that both mechanisms mature postnatally and involve inhibitory mechanisms of regulation.

In adults gastrin generally causes an increase in overall motor activity (12). Previous investigations have shown that fasting plasma gastrin concentrations increase with enteral feeding experience $(4,16,17)$ and that the postprandial release of gastrin is present within the first few postnatal weeks $(8,16)$. In our current study, there is a temporal relationship of motor quiescence and plasma gastrin in the neonatal gut. Duration of motor quiescence and plasma concentrations of gastrin are initially low during fasting, and they both increase with postnatal age. Because plasma gastrin is associated with an increase rather than a decrease in motor activity in the adult as well as the preterm infant $(12,18,19)$, it is unlikely that the temporal relationship of motor quiescence and plasma gastrin is causal. Gastrin may play an important role in the growth and function of neonatal gastrointestinal mucosa (19), and its postnatal change may be reflective of other changes occurring in the newborn gut that are independent of motor function. However, as such, its presence may serve as an additional marker for neonatal gut maturation.

Unlike gastrin, which excites motor activity, PYY inhibits motor activity. It is released in the distal small intestine in response to the presence of fats and is thought to serve as the "ileal brake" (20). As in human infants, plasma concentrations of PYY in neonatal canines were initially higher than values reported for adults and concentrations increased even more with postnatal age $(4,5)$. Infant formulas and breast milk contain high fat content and, as such, may induce the high plasma concentrations reported here. Alternatively, high plasma concentrations of PYY are seen in adults who have gastrointestinal diseases that appear to result in fat malabsorption (21). Thus, one could speculate that the delivery of a high fat load to the distal small intestine in the neonate triggers the release of PYY, which, in turn, inhibits motor activity.

Considerable neonatal morbidity results from attempts to provide preterm infants enteral nutrition. If the mechanisms that regulate the postnatal change in neonatal motor activity patterns could be elucidated, nutritional or pharmacologic management might potentially be useful to promote maturation of intestinal motor activity patterns and thus reduce neonatal morbidity. Although several studies have characterized the postnatal maturation of preterm motor activity and the effects enteral nutrients have on its postnatal maturation $(4,5)$, ethical considerations limit studies in humans to study the mechanisms whereby these maturational changes are regulated. Hence, there is need for an animal model that reliably reproduces the postnatal maturation of the preterm human infant. Although pigs have been used extensively for studies concerning nutrition and intestinal mucosal maturation, their motor activity patterns are not similar to those in humans, in that fasting motor activity is not present unless prolonged periods of starvation occur (22). Recent studies have also shown that neonatal pigs fail to release gastrin in response to feeding (23). The current study shows that these two aspects of functional maturation of humans and canine intestine are similar. Moreover, we have also shown that the maturation of the enteric nervous system parallels maturation of motor activity patterns in newborn dog (24). Thus, three aspects of canine neonatal intestinal function change postnatally in concert: motor activity, the release of peptides, and the appearance of neurochemical markers of the ENS.

\section{REFERENCES}

1. Siegel M, Lebenthal E 1981 Development of gastrointestinal motility and gastric emptying during fetal and newborn periods. In: Lebenthal E (ed) Human Gastrointestinal Development. Raven Press, Philadelphia, pp 277-298

2. Amarnath RP, Berseth CL, Malagelada JR, Perrault J, Abell TL, Hoffman AD 1989 Postnatal maturation of small intestinal motility in preterm and term infants. $J$ Gastrointest Motil 1:38-143

3. Bisset WM, Watt JB, Rivers RPA, Milla PJ 1988 Ontogeny of fasting small intestinal motor activity in the human infant. Gut 29:483-88

4. Berseth CL 1992 Effect of early feeding on maturation of the preterm infant's small intestine. J Pediatr 120:947-53

5. Berseth CL, Nordyke C 1993 Enteral nutrients promote postnatal maturation of intestinal motor activity in preterm infants. Am J Physiol 246:61046-61051

6. Bueno L, Ruckebusch Y 1979 Perinatal development of intestinal myoelectrical activity in dogs and sheep. Am J Physiol 237:E61-E67

7. Berseth CL, Michener SR, Nordyke CK, Go VLW 1990 Postpartum changes in pattern of gastrointestinal regulatory peptides in human milk. Am J Clin Nutr 51:985-990

8. Berseth CL, Nordyke CK, Valdes MG, Furlow BL, Go VLW 1992 Responses of gastrointestinal peptides and motor activity to milk and water feedings in preterm and term infants. Pediatr Res 31:587-590

9. Malloy MH, Morris FH, Denson SE, Weisbrodt NW, Lichtenberger LM, Adcock EW III 1979 Neonatal gastric motility in dogs: Maturation and response to pentagastrin. Am J Physiol 236:E562-E566

10. Berseth CL 1989 Gestational evolution of small intestine motility in preterm and term infants. J Pediatr 115:646-651

11. Koenig WJ, Amarnath RP, Hench V, Berseth CL 1995 Manometrics for preterm and term infants: A new tool for old questions. Pediatrics 95:203-206 
12. Malagelada J-R, Camilleri M, Stangchellini V 1986 Manometric Diagnosis of Gastrointestinal Motility Disorders. Thieme, New York

13. Berseth CL, Baker J 1993 Inhibitory regulation of intestinal motor patterns change postnatally in neonatal humans and canines. J Gastrointest Motil 5:180(abstr)

14. Berseth CL, Nordyke CK 1992 Manometry can predict feeding readiness in preterm infants. Gastroenterology 103:1523-1526

15. Roza Z, Berseth CL, Szurzewski JH 1992 Capsaicin sensitive afferent nerves influence postnatal development of canine duodenal motor activity. Gastroenterology 101, A576

16. Lucas A, Bloom SR, Aynsley-Green A 1982 Postnatal surges in plasma gut hormones in term and preterm infants. Biol Neonate 41:63-67

17. Meetze W, Valentine C, McGuigan J, Conlon M, Sacks N, Neu J 1992 Gastrointes* tinal priming prior to full enteral nutrition in very low birth weight infants. Pediatr Gastroenterol 15:163-70

18. Berseth CL, Go VLW 1990 Correlation of gastrointestinal hormones with maturation of neonatal small intestinal motility. Gastroenterology 97:A178
19. Lichtenberger L 1984 A search for the origin of neonatal hypergastrinemia. J Pediatr Gastroenterol Nutr 3:161-166

20. Holgate AM, Read NW 1985 Effect of ileal infusion of intralipid on gastrointestinal transit, ileal flow rate, and carbohydrate absorption in humans after ingestion of a liquid meal. Gastroenterology 88:1005-1011

21. Adrian TE, Savage AP, Bacarese-Hamilton AJ, Wolfe K, Besterman HS, Bloom SR 1986 Peptide YY abnormalities in gastrointestinal diseases. Gastroenterology 90:379-84

22. Ruckebusch Y, Bueno L The effect of feeding on the motility of the stomach and the small intestine in the pig. $1976 \mathrm{Br}$ J Nutr 35:397-405

23. Szabo JS, Rayford PL, Hooyschuur E Gastrointestinal (GI) hormones in the developing neonatal piglet. Pediatr Res $31: 118 \mathrm{~A}$

24. Berseth CL, DeGiorgio R, Nelson DK, Sternini C, Go VLW 1992 Functional and neurochemical ontogeny of the enteric nervous system (ENS) in neonatal canine smal intestine. Regul Pept 40:112 